Estudios Románicos, Volumen 29, 2020, pp. 331-343

ISSN: 0210-4911

eISSN: $1989-614 \mathrm{X}$

DOI: https://doi.org/10.6018/ER.422501

\title{
L'ESEMPLARITÀ DI TISBE NEL DE MULIERIBUS CLARIS DI BOCCACCIO
}

(Thisbe's exemplarity in Boccaccio's De mulieribus claris)

\author{
Francisco José Rodríguez-Mesa* \\ Universidad de Córdoba
}

\begin{abstract}
Chapter XIII of De mulieribus claris is devoted to the biography of Thisbe. For his narration, Boccaccio uses as his main source the fourth book of Ovid's Metamorphoses although at certain times he modifies the Latin work. For instance, the suffering of Pyramus is no longer equal to that of Thisbe, and the Babylonian maid is the main victim of the love narrated: firstly because of the prohibition of her parents and later for the delay of Pyramus's arrival at the agreed appointment.

Considering all these peculiarities, this paper analyses chapter XIII of the De mulieribus claris and its main character with the purpose of reflecting on their originality and trying to determine if, taking these specificities into account, the Babylonian girl can be considered as an exemplary woman in Boccaccio's work and why.
\end{abstract}

Keywords: Boccaccio; De mulieribus claris; Exemplary women tradition; Thisbe; Pyramus; Women in Medieval literature.

Riassunto: Il capitolo XIII del De mulieribus claris è dedicato alla biografia di Tisbe. Per la sua narrazione, Boccaccio utilizza come fonte principale il quarto libro delle $\mathrm{Me}$ tamorfosi ovidiane sebbene in certi momenti si distacchi dall'opera latina. Ad esempio, la sofferenza di Piramo non è più all'altezza di quella di Tisbe, e la fanciulla babilonese è la principale vittima dell'amore narrato: in primis per la proibizione dei propri genitori e in un secondo momento per la tardività di Piramo nell'arrivare all'appuntamento accordato.

Considerando queste particolarità, questo articolo analizza il capitolo XIII del De mulieribus claris e la sua protagonista con lo scopo di riflettere sull'originalità e di cer-

" Dirección para correspondencia: Francisco José Rodríguez Mesa. Dpto. de Ciencias del Lenguaje. Área de Filología Italiana. Facultad de Filosofía y Letras. Plaza del Cardenal Salazar s/n. 14071 Córdoba [francisco.rodriguez.mesa@uco.es]. 
care di determinare se, tenuto conto di queste specificità, la ragazza babilonese possa considerarsi un personaggio esemplare all'interno della silloge e perché.

Parole chiave: Boccaccio; De mulieribus claris; letteratura esemplare femminile; Tisbe; Piramo; donne nella letteratura medioevale.

Tisbe e il suo amato, Piramo, sono i protagonisti di una delle storie d'amore più largamente coltivate nella mitologia classica e con maggior successo nella letteratura e nelle arti. Le fonti mitografiche danno due versioni diverse del loro tragico amore. La prima di esse che, secondo Grimal (1987: 548), per i propri tratti e la struttura è da considerare come quella più arcaica, narra come entrambi i giovani mantennero rapporti sessuali prima di sposarsi, in modo che Tisbe rimase incinta e, disperata per la situazione, decise di suicidarsi. Piramo, avendo appreso la morte dell'amata, seguì i passi di Tisbe e si tolse la vita. Questa tragedia causò una grande costernazione fra gli dei che, solidali con gli amanti, li unirono per tutta l'eternità grazie alla trasformazione di Piramo nell'omologo fiume di Cilicia e di Tisbe in una sorgente che aumenta la sua portata $^{1}$.

La seconda versione dell'episodio, molto più conosciuta, proviene dalle Metamorfosi ovidiane (IV, 55-166) ${ }^{2}$. Secondo questa narrazione, Piramo e Tisbe erano due giovani babilonesi che crebbero insieme dato che abitavano l'uno accanto all'altro, e che, con il passar del tempo, si innamorarono appassionatamente. Ciononostante, per via dell'opposizione delle loro famiglie al loro amore, furono costretti a tenere segreto il rapporto, cosicché parlavano solo tramite una breccia nel muro che separava le loro case. Queste conversazioni furtive aumentarono la fiamma del loro amore, il che li portò a programmare una fuga notturna per poter stare insieme e parlarsi senza la barriera del muro. Tisbe arrivò per prima al posto concordato, una sorgente nei dintorni della città dove proprio in quel momento saziava la sua sete una leonessa che aveva appena divorato un animale. Davanti a questa situazione, la fanciulla fuggì impaurita senza accorgersi che, nella corsa, aveva perso il proprio velo. La leonessa, dopo essere andata via dalla sorgente e con le fauci ancora insanguinate, si mise a giocare con il velo di Tisbe, che strappò e macchiò di sangue.

Poco dopo, Piramo giunse alla sorgente e, trovando il velo dell'amata stracciato e insanguinato, pensò che essa fosse stata divorata da qualche belva come conseguenza del suo ritardo, per cui, sconsolato, prese il proprio pugnale e si ferì mortalmente al petto. Quando Piramo era già agonizzante, ma ancora vivo, Tisbe uscì dal suo nascondiglio e trovò l'amato sotto un gelso mentre questi esalava gli ultimi respiri. Colpevole per aver

1 Tra tante altre testimonianze, questa versione del mito si può trovare in modo implicito nelle descrizioni geografiche di Senofonte (2002: 85) e Strabone (1954: 353-357), che parlano del fiume Piramo. Narrazioni più estese con riferimenti agli amanti e alla loro fine sono quelle che -fra gli altri- mostrano le opere di Pseudo-Clemente (1993: 5-18), Himerio di Bitinia (1989: 323), Temistio di Bitinia (1995: 14), Nikolaos di Myra (1832: 271) e Nonno di Panopoli (2003: 504-507, 824-825).

2 La versione ovidiana della storia godette di grande fortuna già fra gli autori classici, come dimostrano le narrazioni di Iginio (2000: 141-142), Alcimus (1906: 178), Sant'Agostino (1922: 37), Servio (1961: 68) e Lattanzio Placido (1591: 98). 
provocato questa situazione e disperata per la perdita di Piramo, si tolse la vita con lo stesso pugnale che egli aveva sprofondato nel proprio petto.

Gli dei, commossi dalla forza dell'amore di entrambi, decisero di tingere di nero i frutti del gelso (fino ad allora bianchi) come simbolo del destino che Piramo e Tisbe dovettero affrontare. Allo stesso modo, quando la pira funebre si fu consumata, le ceneri dei due amanti furono depositate in una sola urna affinché riposassero insieme eternamente.

Nonostante le due versioni esistenti, fu quella di impronta ovidiana a godere di fortuna dopo l'Antichità Classica. In effetti, non furono pochi gli autori medievali a raccontare la tragica fine degli amori di Piramo e Tisbe come conseguenza del malinteso provocato dal velo insanguinato. Così, quando Giovanni Boccaccio, nel Filocolo, accennò, per la prima volta nella sua produzione, alla storia degli amanti babilonesi, il mito si poteva dire ben conosciuto fra gli intellettuali del suo tempo ${ }^{3}$. Tuttavia, la timida allusione presente nel primo romanzo ${ }^{4}$ non costituisce che la prima occorrenza della storia nell'opera del certaldese. Circa un decennio dopo, l'autore nomina gli amanti e l'episodio del gelso nella Teseida (VII 62) e nell'Elegia di Madonna Fiammetta (1998b: 68) e fa nuovamente cenno all'argomento nella Comedia delle ninfe fiorentine (XXVI, 36) e nell' Amorosa visione (XXI, 1-12). Ciononostante, solo due decenni dopo, intorno al 1361, Boccaccio riprende la storia e fa diventare Tisbe la protagonista del tredicesimo capitolo del De mulieribus claris, la sua silloge di biografie di donne celebri.

Anche nel caso del $D M C$, dove la vicenda è narrata con dettaglio, la fonte principale è Ovidio, come hanno già dimostrato diversi studiosi (Zaccaria, in Boccaccio 1967: 493; Brown, in Boccaccio 2003: 241; Filosa 2006: 2; Filosa 2012: 95-96), fino al punto che fra i due autori si possono perfino riscontrare coincidenze lessicali5. Tuttavia, tenuto conto dell'ampia presenza di questo episodio nelle opere di Boccaccio, non c'è da meravigliarsi se l'autore aggiunge al racconto delle Metamorfosi dei particolari che fanno sì che la narrazione originale guadagni in originalità, freschezza e attualità. Con le parole di Filosa, "La tragica storia di Tisbe e Piramo, staccata dalla fonte ovidiana, vive ormai di vita propria nella mente dell'autore certaldese, arricchendosi di altro materiale letterario, in tal caso dantesco, pur rimanendo sempre sé stessa nella sua unicità tematica" ${ }^{6}(2012$ : 110).

3 Tra le testimonianze anteriori a quelle del certaldese, sempre secondo la versione ovidiana, si possono annoverare Arnolfo d'Orleans (Ghisaberti 1932: 53), Giovanni di Garlandia (Ghisaberti 1933a: 116), Matteo di Vendôme (1982: 164-169), Gervasio di Melkley (Faral 1924: 331-335), il compilatore della Gesta Romanorum (Oesterley 1872: 633), Dante (Purg. XXVII, 35-42; XXXIII, 69) o Giovanni del Virgilio (Ghisaberti 1933b: 54).

4 Questa prima allusione si riduce a una sola frase «Per quale altra cagione diventò il gelso vermiglio, se per l'ardente fiamma costretta, la quale prese più forza ne' due amanti costretti di non vedersi?» (Boccaccio 1998a: 76).

5 Per i particolari sulla lingua del $D M C$, si veda l'ormai classico Zaccaria (1965).

6 Filosa non è la sola autrice a voler vedere nei brani del Purgatorio dantesco che alludono alla storia d'amore tra Piramo e Tisbe (vedi nota 3) una sorta di influenza per la biografia del $D M C$, anche Kolsky affermò che "The fact that Dante includes mention of the episode in Purgatorio [...] may have influenced Boccaccio's decision to insert this [...] story in the De mulieribus" (2003: 33). Tuttavia, a nostro avviso, è molto azzardato considerare le parole del Purgatorio una qualsiasi fonte per Boccaccio, poiché i brevi cenni danteschi al mito, derivanti chiaramente da Ovidio, menzionano ben due volte il gelso (Purg. XXVII, 39; XXXIII, 69) e questo è un elemento che -come si vedrà- il certaldese, per rispetto alla tradizione evemeristica, decide di eliminare nel suo racconto. 
In questo panorama, stupisce la scarsa attenzione che gli studiosi del certaldese e del $D M C$ hanno dedicato alla figura di Tisbe e al suo ruolo all'interno della silloge muliebre. Così, uno dei principali lavori sulle eroine in Boccaccio (Franklin 2006) ignora totalmente Tisbe, mentre lo spazio che essa occupa in quello che si può considerare il pioniere fra gli studi del DMC (Müller 1992) non si può definire che marginale. Nello stesso modo, non si può dire - a nostro avviso- che gli studi del nuovo millennio sul catalogo di donne famose del certaldese siano stati di grande aiuto per l'analisi del capitolo XIII e del ruolo che la sua protagonista ha nell'insieme della raccolta. In effetti, sebbene ci siano diverse allusioni commentate, neanche lo studio di Filosa (2012) mostra un approccio approfondito della biografia di Tisbe. Solo Kolsky (2003: 33-36) consacra alcune pagine del suo studio sul $D M C$ a discorrere con più dettaglio sull'episodio della giovane babilonese, anche se -dal nostro punto di vista- lo fa da una prospettiva che incide troppo sulla dicotomia $D M C$ vs. Decameron per quanto riguarda le strategie narrative, i temi e la caratterizzazione delle donne presenti in entrambe le opere e lascia da parte l'analisi della vera e propria presenza di Tisbe nel catalogo muliebre.

Tenuto conto di questi fatti, in questo studio verrà analizzato il ruolo di Tisbe da una duplice prospettiva: sia per quanto riguarda la sua funzione come protagonista della tredicesima biografia (senza dimenticare che essa proviene dalla fonte ovidiana), sia rispetto alla sua funzione -vale a dire, al suo valore esemplare-nell'insieme del $D M C$.

Che quella di Tisbe non fosse per Boccaccio una biografia qualunque si può provare analizzando le diverse posizioni che, nell'architettura globale della silloge, il capitolo occupò nelle varie fasi di stesura della raccolta ${ }^{7}$. Infatti, considerando i manoscritti denominati $\mathrm{Vu}, \mathrm{L}$ e $\mathrm{L}_{1}(=\text { volgata })^{8}$, la posizione della biografia della fanciulla babilonese subisce importanti modifiche, dato che passa dall'ultimo posto in $\mathrm{Vu}$ al penultimo in $\mathrm{L}^{9}$ e alla sua posizione definitiva nel tredicesimo capitolo in $\mathrm{L}_{1}{ }^{10}$. Questa posizione conclusiva nelle prime stesure dell'opera dimostra che, prima di affidarsi a criteri cronologici ${ }^{11}$, il certaldese concepì la vita di Tisbe come quella che avrebbe segnato la fine del percorso narrativo del $D M C$ che-ricordiamoci-si apre proprio con la biografia di Eva.

È vero che, come già sottolineato, Ovidio rimane la fonte principale per la biografia di Tisbe nel $D M C$, ma fra la storia del quarto libro delle Metamorfosi e quella narrata

$7 \quad$ Le fasi di composizione del $D M C$ sono state stabilite con una chiarezza che ammette pochi dubbi. Nello studio che pose le basi di quest'argomento, Ricci (1959: 132-134) stabilì sette fasi redazionali mentre, pochi anni dopo, Zaccaria (1963: 293) ne distinse nove. Per ulteriori informazioni sulla datazione e le modifiche che la silloge presenta nei manoscritti che la tramandano, vedi anche Zappacosta (1973), Branca-Zaccaria (1996) e Nuvoloni (2003).

8 Come al solito negli studi sulla tradizione manoscritta dell'opera, utilizziamo la denominazione codicologica stabilita da Branca (1958: 92-97).

9 Bisogna sottolineare che sia in Vu sia in L l'episodio di Tisbe occupa la stessa posizione (104), sebbene in L Boccaccio aggiunga un capitolo aggiuntivo, titolato "De feminis nostri temporis", per chiudere l'opera. Quest'ultimo capitolo diverrà, in $\mathrm{L}_{1}$, la conclusione alla raccolta.

10 Lo stesso Zaccaria fece riferimento all'importanza di questi cambiamenti, citando la vita di Tisbe come perfetto esempio delle differenze fondamentali nella tradizione codicologica dell'opera (1963: 256).

11 Bisogna ricordare che, in una misura importante, le modifiche che portarono l'autore a concludere la silloge con le vite di Camiola (CV) e della regina Giovanna (CVI) furono di natura propagandistica e non strettamente letterarie (vedi Rodríguez-Mesa 2019). 
da Boccaccio ci sono divergenze significative. Così, la narrazione ovidiana potrebbe essere ridotta al seguente schema:

1. Introduzione: presentazione dei due amanti (vv. 55-60)

2. In città

a. Amor proibito (vv. 60-62)

b. Conversazioni tramite la fessura del muro e piano di fuga (vv. 63-92)

3. Arrivo alla sorgente

a. Tisbe esce di casa, scopre la leonessa e si nasconde (vv. 93-104)

b. Malinteso di Piramo e suicidio (vv. 105-127)

c. Tisbe trova Piramo moribondo (vv. 128-141)

i. Monologo finale (vv. 142-161)

ii. Suicidio (vv. 162-163)

4. Conseguenze delle morti

a. Il gelso cambia colore (v. 165)

b. Le cenere di entrambi riposano nella stessa urna (v. 166)

Mentre la struttura di $D M C$, XIII si potrebbe riassumere $\operatorname{cosi}^{12}$ :

1. Introduzione

a. Presentazione di Tisbe (1)

b. Nascita dell'amore tra Tisbe e Piramo (2)

2. In città

a. Amor proibito (3a)

b. Conversazioni tramite la fessura del muro e piano di fuga (3b-4a)

3. Arrivo alla sorgente

a. Tisbe scopre la leonessa e si nasconde (4b)

b. Malinteso di Piramo e suicidio (5)

c. Tisbe trova Piramo moribondo e si suicida (6-11)

4. Conclusione dell'autore

a. Riflessioni sulla storia (12)

b. Riflessioni generali (13-14)

Come si può osservare, la divergenza che salta in modo più evidente agli occhi del lettore ha a che vedere con il segmento della storia degli amanti che ciascuno dei due testi narra. Così, il testo boccaccesco omette gli avvenimenti accaduti dopo la morte degli amanti -cioè, la spiegazione mitologica del colore dei gelsi ed il fatto che Piramo e Tisbe riposarono insieme per l'eternità- e si chiude con una riflessione morale sulla colpevolezza dei genitori nel cercar di frenare l'amore di gioventù.

È vero che l'elisione dell'elemento fantastico del gelso -conseguenza della compassione divina- apporta un'ulteriore componente di verosimiglianza al racconto del

12 Fra parentesi si fa riferimento alle suddivisioni del testo originale. 
$D M C$, al tempo che consente che la storia confluisca nella corrente evemeristica così presente nei lavori mitografici del certaldese. Allo stesso modo, la costruzione dell'epilogo della biografia in Boccaccio gira attorno a un insieme di elementi morali -quasi con valore precettivo- che cominciano in un modo specifico, e che sono legati alla storia appena narrata:

Quis non compatietur iuvenibus? Quis tam infelici exitui lacrimulam saltem unam non concedet? Saxeus erit. Amarunt pueri: non enim ob hoc infortunium meruere cruentum. Florentis etatis amor crimen est, nec horrendum solutis crimen; in coniugium ire poterat. Peccavit fors pessima et forsan miseri peccavere parentes.

[Chi non proverà pietà per la sorte dei due giovanetti? Chi non verserà almeno una lacrimetta per così tragico destino? Chi la negasse, avrebbe il cuore di pietra. I giovanetti, è vero, si amarono: ma non per questo meritarono morte cruenta. L'amore è un peccato dell'età giovanile, ma non detestabile, almeno per coloro che sono liberi da altri vincoli; e il loro avrebbe potuto sboccare nel matrimonio. Il vero peccato fu quello del perfido destino e forse dei loro disgraziati genitori]. (Boccaccio 1967: 70-71)

Subito dopo, quest'approccio cambia per diventare molto più universale:

Sensim quippe frenandi sunt iuvenum impetus, ne, dum repentino obice illis obsistere volumus, desperantes in precipitium inpellamus. Immoderati vigoris est cupidinis passio et adolescentium fere pestis et comune flagitium, in quibus edepol patienti animo tolleranda est, quoniam sic rerum volente natura fit, ut scilicet dum etate valemus, ultro inclinemur in prolem, ne humanum genus in defectum corruat, si coitus differantur in senium.

[È vero che bisogna frenare gli impulsi dei giovanetti, ma gradatamente, per non spingerli disperati al precipizio, proprio mentre si cerca di resistere con improvvisi ostacoli al loro amore. La passione dei sensi è per se stessa smodata ed è malanno e quasi peste comune a tutti i giovani. Ma bisogna proprio in essi saperla tollerare con pazienza. La natura stessa vuole che, fin che siamo giovani, sentiamo spontaneo lo stimolo a procreare; in modo che la stirpe umana non si estingua, se i congiungimenti carnali siano differiti al tempo della vecchiaia.] (Boccaccio 1967: 70-71)

Con questi elementi Boccaccio sottolinea la componente didattica a cui aveva già accennato nel prologo della raccolta, secondo la quale il certaldese concepisce la propria opera -e ci si consenta di usare la terminologia petrarchesca- come un lavoro con applicazione "ad vitam"13. Quest'elemento morale si trova qua secondo una palese modalità oraziana, ed infatti il "docere et delectare" è sempre stato uno dei tratti più frequentemente sottolineati nella vita di Tisbe. Come ha affermato Filosa (2006: 2) e più di un secolo prima di lei dichiarò Hortis, il delectare nell'opera è raggiunto

13 Secondo Petrarca, lo studio va orientato "magis [...] ad vitam [...] quam eloquentiam" (Familiares, I, III, 4). Per approfondire in quest'atteggiamento pratico dell'attività umanistica si veda Rico (2002: 59-61). 
E col racconto 'copioso', particolareggiato, d'avvenimenti che destano la curiosità de' lettori, e col racconto di fatti che mettono l'animo in commozione, della quale ben sapeva il Boccaccio che erano particolarmente vaghe le 'sue care donne'. [...] Fra le donne famose merita certamente di essere ricordata l'infelice Tisbe; e il Boccaccio ne racconta la lagrimosa storia (Hortis 1877: 13)

Oltre agli elementi strutturali ci sono altre divergenze fra il testo del $D M C$ e la fonte ovidiana della vita di Tisbe, e queste spiccano in modo particolare perché comportano differenze nella caratterizzazione dei personaggi.

La prima di queste divergenze riguarda il vero motore dal quale la narrazione prende spunto, e cioè, i motivi per i quali il rapporto fra i due giovani viene vietato. In questo senso, Boccaccio afferma che "sane, cum iam grandiuscula fieret Tisbes, a parentibus in futuros hymeneos domi detineri cepta est" ["Quando Tisbe fu grandicella, i genitori cominciarono a tenerla in casa, coll'intenzione di maritarla"] (Boccaccio 1967: 66-67); vale a dire, si tratta di una decisione che presero i genitori di Tisbe, senza che nel $D M C$ ci sia un qualsiasi riferimento a quelli di Piramo. Ovidio, da parte sua, osserva che "...Taedae quoque iure coissent, / sed vetuere patres..." (Met. IV, 60-61) ["si sarebbero uniti in legittime nozze, se non fosse che i padri lo proibirono" (Ovidio 1994: 135)], ritenendo responsabili i genitori di entrambi i giovani. A nostro avviso, quest'allusione confluisce nell'invocazione finale della Tisbe ovidiana, che dichiara

Hoc tamen amborum verbis estote rogati,

o multum miseri meus illique parentes,

ut quos certus amor, quos hora novissima iunxit,

componi tumulo non invideatis eodem. (Met. IV, 154-157)

["Questa preghiera tuttavia vi rivolgiamo ambedue, o infelicissimi padre mio e padre suo, di non negarci di essere composti in uno stesso sepolcro, noi che siamo stati uniti nell'estremo istante" (Ovidio 1994: 141)]

Il punto di coincidenza fra questi due brani è che, a nostro parere, sembrano indicare che, nelle Metamorfosi, ci sia una rivalità familiare che serve da sfondo alla storia di Piramo e Tisbe e che giustifica che il divieto al rapporto fra i giovani venga da entrambe le famiglie.

Il secondo degli elementi diegetici che separa le versioni della storia di Tisbe in Boccaccio e Ovidio ha a che fare con la fuga che i giovani scelgono per poter incontrarsi. Il certaldese descrive le intenzioni degli amanti affermando "Tandem, excrescente incendio, de fuga inivere consilium, statuentes ut nocte sequenti, quam primum quis posset suos fallere, domos exiret; et seinvicem, si quis primus evaderet, in nemus civitati proximum abiens" ["Finalmente, quando l'incendio d'amore divampò, stabilirono un piano di fuga, coll'intesa che, la notte seguente, non appena uno potesse eludere la vigilanza dei suoi, uscisse di casa e si recasse nel bosco fuori della città"] (Boccaccio 1967: 66-69). Da queste parole si potrebbe evincere che 
l'incontro notturno che finisce in modo funesto è inizialmente concepito come una fuga definitiva dalle case paterne da parte dei giovani e non semplicemente come un incontro puntuale.

Ovidio, invece, non è così chiaro nella sua descrizione:

...tum murmure parvo

multa prius questi statuunt, ut nocte silenti

fallere custodes foribusque excedere temptent,

cumque domo exierint, urbis quoque tecta relinquant,

neve sit errandum lato spatiantibus arvo,

conveniant ad busta Nini lateantque sub umbra

arboris... (Met. IV, 83-89)

["Allora, bisbigliando sommessamente, dopo essersi a lungo lamentati, stabilirono di eludere la vigilanza e di tentar di uscire di casa nel silenzio della notte, e una volta fuori, di lasciare anche l'abitato e, per non sperdersi vagando per l'aperta campagna, d'incontrarsi al sepolcro di Nino e nascondersi al buio sotto l'albero" (Ovidio 1994: 137)]

I termini del poeta latino e, in modo speciale, i versi $88-89$, sembrano accennare al fatto che lo scopo dell'uscita dalla casa familiare altro non fosse che quello di trascorrere la notte insieme "sub umbra arboris", senza escludere che i giovani potessero rincasare prima dell'alba.

Probabilmente questo elemento della narrazione delle Metamorfosi è da collegare alle limitazioni delle quali gli innamorati si lamentano ripetutamente davanti al muro: esso costituisce una barriera insormontabile per il contatto fisico così apertamente voluto e così apertamente desiderato dalla Tisbe e dal Piramo ovidiani, come testimoniano diversi brani della storia: "Invide' dicebant 'paries, quid amantibus obstas? / Quantum erat, ut sineres toto nos corpore iungi / aut, hoc si nimium est, vel ad oscula danda pateres!" (Met. IV, 73-75) ["Dicevano 'muro cattivo, perché ostacoli il nostro amore? Quanto sarebbe meglio se ci permettessi di unirci con tutto il corpo, o, se questo è troppo, almeno ti aprissi quel tanto che basta per darsi dei baci!" (Ovidio 1994: 137)]; "Talia diversa nequiquam sede locuti / sub noctem dixere 'Vale' partique dedere / oscula quisque suae non pervenientia contra" (Met. IV, 78-80) ["Pronunciate inutilmente frasi di questo genere, restando divisi, una sera si salutarono e ciascuno dette alla sua parte del muro dei baci che non arrivarono di là" (Ovidio 1994: 137)].

Forse, come conseguenza della moralità dell'epoca e dello scopo didattico del $D M C$, gli amanti boccacceschi si mostrano molto più misurati su quest'aspetto, poiché l'opera non mostra alcun lamento riguardo al muro come barriera che impedisce il contatto fisico fra gli innamorati e la narrazione delle parole che essi scambiano -in opposizione all'accesa passione ovidiana- si limita ad indicare che "ad quam dum clam convenissent sepius et, consuetudine paululum colloquendo, pariete etiam obice, quo minus erubescebant, ampliassent exprimendi affectiones suas licentiam, sepe suspiria lacrimas fervores desideria et passiones omnes aperiebant vias" ["Spesso furtivamente le si av- 
vicinavano [alla fessura] e si parlavano alquanto attraverso la parete divisoria e, senza vergogna, accentuavano le espressioni affettuose, spesso anche dando libero sfogo a sospiri e lagrime, desideri ardenti e passioni”] (Boccaccio 1967: 66-67).

Queste parole contrastano con la lettura che di questa biografia fa Kolsky nell'affermare che Tisbe è una protagonista atipica poiché "Unmarried women in the De mulieribus claris are not often involved in illicit sexual activity" (2003: 33). A nostro parere, la storia della fanciulla non è affatto atipica giacché Boccaccio, a differenza della fonte ovidiana, non parla di passione carnale ${ }^{14}$, ma esalta la costanza nell'amore nonostante le difficoltà. Anzi, nella reprensione all'atteggiamento dei genitori che serve come conclusione al racconto, l'autore afferma chiaramente che "florentis etatis amor crimen est, nec horrendum solutis crimen" ["l'amore è un peccato dell'età giovanile, ma non detestabile, almeno per coloro che sono liberi da altri vincoli"] (Boccaccio 1967: 70-71). Detto in altri termini, si potrebbe dire che, mentre per Ovidio Piramo e Tisbe sono due amanti, per Boccaccio sono due innamorati.

Nonostante queste particolarità, che fanno sì che il testo boccaccesco sembri meno audace dal punto di vista morale rispetto della fonte ovidiana, c'è un piccolo dettaglio in cui l'episodio del $D M C$ sembra osare più di quanto non facciano le $M e$ tamorfosi, e ha a che vedere con la configurazione di Tisbe come personaggio, principalmente con i tratti che la contraddistinguono come amante. È già stato segnalato come, in opposizione con la fonte ovidiana, dove entrambe le famiglie sono contrarie agli amori di Piramo e Tisbe, in Boccaccio solo alla fanciulla viene vietata la realizzazione del proprio desiderio, dato che i genitori cercano di trovarle un marito adeguato -che ovviamente non è Piramo-. Questa modifica, in apparenza banale, fa sì che, secondo la biografia del $D M C$, Tisbe sia il personaggio che si espone ad un rischio maggiore o, anzi, l'unico personaggio che si espone ad un vero e proprio rischio mantenendosi fedele e costante ai propri sentimenti e perseguendo la materializzazione delle proprie passioni.

In questo senso e in consonanza con l'ardore con cui è pronta ad abbandonarsi a Piramo, Tisbe è la prima a fuggire da casa sua la notte dell'appuntamento, il che il certaldese lo attribuisce al fatto che lei fosse "ardentior forte" ["forse più ardente"] (Boccaccio 1967: 68-69) di Piramo. Il ritardo di Piramo nel giungere al luogo dell'appuntamento manca di giustificazione interna nel contesto creato dal $D M C$, poiché -dato che non doveva sconfiggere alcun ostacolo familiare per uscire di casa- potrebbe essere interpretato come una mostra di disinteresse o di trascuratezza e questo ritardo, non dobbiamo dimenticarcene, è il principale responsabile della sanguinosa fine della storia, ancora più importante del divieto paterno per quanto riguarda Tisbe.

Questa nuova ottica dalla quale Boccaccio descrive gli avvenimenti contribuisce a conferire all'episodio due elementi dei quali era totalmente privo nelle versioni di ispi-

14 Il solo momento in cui il certaldese allude al desiderio sessuale nel capitolo dedicato a Tisbe è nella reprensione finale, dove fa riferimento all'atteggiamento generale dei giovani (e non a quello dei protagonisti della biografia) nell'indicare "Immoderati vigoris est cupidinis passio et adolescentium fere pestis et comune flagitium" ["La passione dei sensi è per se stessa smodata ed è malanno e quasi peste comune a tutti i giovani”] (Boccaccio 1967: 70-71). 
razione classica ${ }^{15}$. Il primo di essi radica nel fatto che il $D M C$ attribuisce un protagonismo assoluto al personaggio femminile. In effetti, è Tisbe con le sue circostanze familiari e con il suo ardore e la sua iniziativa nell'agire il principale motore della trama. In questo contesto, Piramo resta confinato ad un secondo piano e la sua passività quasi costante si tramuta in azione solo quando, dopo la scoperta del velo strappato e insanguinato dell'amata, decide di togliersi la vita. Ciononostante, a differenza dei termini con cui il certaldese descrive il suicidio di Tisbe, Piramo non si uccide per amore, bensì per il senso di colpa:

Dumque per silentia noctis intentus comperisset laceratum cruentumque pallium Tisbis, ratus eam a belua devoratam, plangore plurimo locum complevit, se miserum incusans quoniam dilectissime virgini seve mortis causam ipse dedisset; et aspernans de cetero vitam, exerto, quem gesserat, gladio, moribundus secus fontem pectori impegit suo.

["Quando, tutto teso nel silenzio notturno, trovò il mantello di Tisbe lacero e insanguinato, credette che la fanciulla fosse stata sbranata dalla belva. Allora riempì quel luogo del suo pianto, accusandosi di aver dato, egli stesso, infelice, morte crudele all'amatissima donzella. Disprezzando per il resto la vita, sguainò la spada, che portava seco, e l'affondò nel petto, deciso a morire presso la fonte".] (Boccaccio 1967: 68-69)

Dunque, si potrebbe dire che Boccaccio trasforma la storia di Piramo e Tisbe nella storia di Tisbe.

La seconda innovazione della narrazione del $D M C$ è intimamente legata alla precedente e si riferisce ad una virtù che in Tisbe viene sottolineata in un modo speciale: la costanza. Risulta palese dopo quest'esposizione che l'amore costante è la principale qualità della fanciulla boccaccesca. Questa è la fonte dalla quale sembrano sorgere le forze della giovane per prendere le tre decisioni che mettono in moto la trama: restare fedele al proprio amore per Piramo nonostante il divieto dei genitori, fuggire da casa e togliersi la vita.

È vero che le riflessioni messe come conclusione alla biografia puntano più verso la disapprovazione dell'atteggiamento paterno che non verso la celebrazione delle virtù di Tisbe ma, a nostro avviso, questa qualità si mostra con un'intensità tale nella protagonista che questa biografia XIII si potrebbe -e si dovrebbe- collocare in un'orbita simile a quelle che sono state tradizionalmente considerate dalla critica come esempi di amore coniugale, ad esempio, Ipermestra (XIV), Argia (XXIX), le spose dei Menii (XXXI), Penelope (XL), Lavinia (XLI), Artemisia (LVII), Terza Emilia (LXXIV), Ipsicratea (LXXVIII), le spose dei Cimbri (LXXX), Giulia, figlia di Giulio Cesare (LXXXI), Porzia (LXXXII), Curia (LXXXIII), Sulpicia (LXXXV), Pompea Paolina (XCIV) e Triaria (XCVI).

Come indica Filosa (2012: 184-187), tutte queste donne sono ricordate da Boccaccio per le loro virtù relative all'amore coniugale e, quindi, rappresentano un esempio positivo all'in-

15 Basti paragonare questi motivi della narrazione del $D M C$ con gli analoghi di autori quali Servio o Lattanzio Placido. 
terno dell'opera ${ }^{16}$. È vero che Piramo e Tisbe non erano sposati, come lo erano le donne appena elencate con i loro mariti e, quindi, da un punto di vista strettamente lessicale non si potrebbe qualificare la fanciulla babilonese come esempio di amore coniugale, ma nonostante la fermezza del suo carattere, che si mostra lungo tutta la biografia, essa viene considerata semplicemente come un personaggio "neutro" secondo la suddetta classificazione (Filosa 2012: 184) e di Tisbe si sottolinea meramente il fatto che si ricorda per un amore infelice. Or bene, è vero che quest'etichetta è perfettamente applicabile alla Tisbe della tradizione mitografica classica che ha il quarto libro delle Metamorfosi quale paradigma, ma, tenuto conto delle differenze fra questo testo e il $D M C$ che abbiamo evidenziato in questo studio, non riteniamo la denominazione molto adeguata per la versione boccaccesca.

In effetti, è già stato detto che Boccaccio trasforma il racconto ovidiano di Piramo e Tisbe in un episodio di cui Tisbe è protagonista esclusiva e in cui Piramo altro non è che il collaboratore necessario affinché la fanciulla possa esibire la propria costanza e la propria virtù dell'anima. È vero che entrambi sono vittime della funesta fine, ma mentre Piramo muore per una situazione che egli stesso e la sua apparente trascuratezza sembrano aver causato, la morte di Tisbe è la prova ultima e definitiva della sua costanza e del suo amore immutabile.

Bisogna anche ricordare, per concludere, che con queste modifiche della fonte ovidiana, Boccaccio riesce a costruire un testo molto più moderno e molto più ricco di sfumature, soprattutto per quanto riguarda la profondità psicologica di Tisbe. Allo stesso tempo, queste manipolazioni fanno sì che la biografia XIII diventi, ancora una volta se si considera l'insieme dell'opera del certaldese, un testo in cui la virtù e la forza femminili vengono esaltate in un contesto contrassegnato dall'avversità.

\section{BIBLIOGRAFIA}

ALCIMUS (1906): Anthologia Latina. Leipzig: Teubner.

BOCCACCIO, Giovanni (1964): Comedia delle ninfe fiorentine, a cura di A. E. Quaglio. Milano: Mondadori.

(1967): De mulieribus claris, a cura di V. Zaccaria. Milano: Mondadori.

(1974): Amorosa visione, a cura di V. Branca. Milano: Mondadori.

(1992): Teseida delle nozze d'Emilia, a cura di A. Limentani. Milano: Mondadori.

(1998a): Filocolo, a cura di A. E. Quaglio. Milano: Mondadori.

(1998b): Elegia di Madonna Fiammetta, a cura di C. Delcorno. Milano: Mondadori.

(2003): Famous Women, trad. V. Brown. Cambridge: Harvard University Press.

BRANCA, Vittore (1958): Tradizione delle opere di Giovanni Boccaccio. Roma: Edizioni di Storia e Letteratura.

16 L'unica eccezione ne è Triaria, moglie di Lucio Vitellio (XCVI), nella cui vita si descrive anche la ferocia del suo carattere, il che suppone un esempio negativo. 
BRANCA, Vittore; ZACCARIA, Vittorio (1996): "Un altro codice del De mulieribus claris del Boccaccio". Studi sul Boccaccio. 24: 3-6.

FARAL, Edmond (1924): Les arts poétiques du XII et du XIII siècle. Paris: Champion.

FILOSA, Elsa (2006): "Intertestualità tra Decameron e De mulieribus claris: La tragica storia di Tisbe e Piramo". Heliotropia, an Online Journal of Research to Boccaccio Scholars. Vol. 3.1.1: 1-9.

(2012): Tre studi sul "De mulieribus claris". Milano: LED.

FRANKLIN, Margaret (2006): Boccaccio's Heroines. Power and Virtue in Renaissance Society. Burlington: Ashgate.

GHISABERTI, Fausto (1932): Arnolfo d'Orleans: un cultore di Ovidio nel secolo XII. Firenze: Hoepli.

(1933a): Giovanni di Garlandia, Integumenta Ovidii, poemetto inedito del secolo $X I I I$. Messina: Principato.

(1933b): Giovanni del Virgilio, espositore delle "Metamorfosi”. Firenze: Olschki.

GRIMAL, Pierre (1987): Dizionario di mitologia greca e romana. Brescia: Paideia.

HIMERIO DI BITINA (1989): Orationes. Cambridge: Harvard University Press.

HORTIS, Attilio (1877): Le donne famose descritte da Giovanni Boccaccio. Trieste: Stabilimento art. Tip. G. Caprin.

IGINIO (2000): I miti. Milano: Adelphi.

KOLSKY, Stephen D. (2003): The Genealogy of Women. Studies in Boccaccio's "De mulieribus claris". New York: Peter Lang.

LATTANZIO PLACIDO (1591): Narrationes fabularum Ovidianarum. Anversa: Officina Plantiniana.

MATTEO DI VENDÔME (1982): Piramus et Tisbe di Mathei Vindocinensis Opera, a cura di F. Munari. Bologna: Edizioni di Storia e Letteratura.

MÜLLER, Ricarda (1992): Ein Frauenbuch des frühen Humanismus. Untersuchungen zu Boccaccios 'De mulieribus claris'. Stuttgart: Franz Steiner Verlag.

NIKOLAOS DI MYRA (1832): Progymnasmata. Tubingen: Cotta.

NONNO DI PANOPOLI (2003): Le Dionisiache. Milano: Rizzoli.

NUVOLONI, Laura (2003): "De mulieribus claris: un frammento". Studi sul Boccaccio. 31: 23-26.

OESTERLEY, Hermann (1872): Gesta Romanorum. Berlin: Weidmannsche Buchhandlung.

OVIDIO (1994): Metamorfosi. Milano: Einaudi.

PSEUDO-CLEMENTE (1993): Recognitiones. Roma: Città nuova editrice.

RICCI, Pier Giorgio (1959): "Studi sulle opere latine e volgari del Boccaccio", Rinascimento. X, I: 1-21.

RICO, Francisco (2002): El sueño del humanismo. De Petrarca a Erasmo. Barcelona: Destino.

RODRÍGUEZ-MESA, Francisco José (2019): “'Singular decus ytalicum’: la biografia di Giovanna di Napoli nel De mulieribus claris". Estudios románicos. 28: 361373. 
SANT'AGOSTINO (1922): De ordine. Leipzig: Corpus Scriptorum Eccleasiasticorum Latinorum.

SENOFONTE (2002): Anabasi. Torino: UTET.

SERVIO (1961): Servii Grammatici qui feruntur in Vergilii Bucolica et Georgica commentarii. Hildesheim: Georg Olms.

STRABONE (1954): The Geography of Strabo. Cambridge: Harvard University Press. TEMISTIO DI BITINA (1995): Discorsi. Torino: UTET.

ZACCARIA, Vittorio (1963): "Le fasi redazionali del De mulieribus claris". Studi sul Boccaccio. Vol. I: 252-332.

(1965): "Appunti sul latino del Boccaccio nel De mulieribus claris". Studi sul Boccaccio. Vol. III: 229-246.

ZAPPACOSTA, Guglielmo (1973): "Per il testo del De mulieribus claris. I. Il Cod. Laur. Pluteo XC sup. 98 e il testo dell'opera”. Studi sul Boccaccio. 7: 239-245.

\section{PERFIL ACADÉMICO-PROFESIONAL}

Profesor Ayudante Doctor de Filología Italiana en la Universidad de Córdoba. Su investigación se ha centrado en diversos aspectos de la literatura italiana medieval y renacentista, como el análisis de la lírica petrarquista y su difusión, en particular en el sur de la península italiana durante el Quattrocento (Giannantonio de Petruciis, Rustico Romano, el «Cansonero» del conde de Popoli), o Boccaccio y la tipología de las formas narrativas breves. Asimismo, se ha ocupado del papel de la mujer en algunas obras italianas del Trecento, principalmente en Boccaccio (Decameron, De mulieribus claris) y Petrarca (De insigni obedientia et fide uxoria, Rerum vulgarium fragmenta). En los últimos años, ha impartido diversos seminarios en calidad de profesor visitante en varias universidades extranjeras, como la Università La Sapienza di Roma, la Università degli Studi di Udine o la Universidad Nacional Autónoma de México, y ha sido nombrado Investigador Asociado de la Biblioteca Nacional de Uruguay.

Fecha de recepción: 09/04/2020

Fecha de aceptación: 16/05/2020 